\title{
Upregulation of Shh and Ptc1 in hyperoxia-induced acute lung injury in neonatal rats
}

\author{
HONGXING DANG ${ }^{1,2}$, SHAOHUA WANG ${ }^{2,3}$, LIN YANG $^{1,2}$, FANG FANG $^{1,2}$ and FENG XU ${ }^{1,2}$ \\ ${ }^{1} \mathrm{PICU}$, Children's Hospital of Chongqing Medical University; ${ }^{2}$ Ministry of Education \\ Key Laboratory of Child Development and Disorders, Yu Zhong, Chongqing 400014; \\ ${ }^{3}$ NICU, Women and Children Health Institute, Futian, Shenzhen, Gangdong 518045, P.R. China
}

Received February 22, 2012; Accepted May 17, 2012

DOI: $10.3892 / \mathrm{mmr} .2012 .929$

\begin{abstract}
The aim of the present study was to observe the expression of sonic hedgehog (Shh) and Ptc signaling molecules in the lungs of newborn rats exposed to prolonged hyperoxia, and to explore the role of the SHH signaling pathway in hyperoxia-induced lung injury. Newborn Sprague-Dawley rat pups were placed in chambers containing room air or oxygen above $95 \%$ for 14 days following birth. The rats were sacrificed after 3 , 7 or 14 days and their lungs were removed. Sections were fixed and subjected to hematoxylin and eosin (H\&E) staining. Shh and Ptc1 were quantitated by immunohistochemistry. The total RNA and protein were also extracted from lung tissue; realtime PCR (RT-PCR) and western blot analysis were utilized to assess the mRNA and protein expression of Shh and Ptc1. H\&E staining demonstrated significant histomorphological changes in the hyperoxia-exposed lungs at 3,7 and 14 days of age. The results of the immunohistochemistry, RT-PCR and western blot analysis demonstrated that the expression of Shh was significantly higher in the hyperoxia-exposed lungs at 3,7 and 14 days, while Ptc1 was significantly elevated at 7 and 14 days. Exposure of the neonatal rat lung to prolonged hyperoxia resulted in acute lung injury and histomorphological changes. Shh and Ptcl were upregulated in a time-dependent manner in the course of hyperoxia-induced lung injury. The SHH signal pathway may be involved in the pathogenesis of hyperoxia-induced lung injury. This is the first evidence that in vivo hyperoxia induces activation of the SHH signal transduction pathway in newborn lung.
\end{abstract}

\section{Introduction}

As an important therapeutic intervention, high supplemental oxygen concentrations are often administered to neonates with

Correspondence to: Professor Feng Xu, PICU, Children's Hospital of Chongqing Medical University and Ministry of Education Key Laboratory of Child Development and Disorders, 136 Zhongshan No. 2 Road, Yu Zhong, Chongqing 400014, P.R. China

E-mail: xufeng9899@yahoo.com.cn

Key words: hyperoxia, acute lung injury, Shh protein, Ptc1 protein, newborn, rat respiratory diseases, including hyaline membrane disease, persistent pulmonary hypertension and others. High concentrations of oxygen are known to cause acute lung injury (ALI) and bronchopulmonary dysplasia (BPD). Prolonged exposure to hyperoxia can also result in severe epithelial and endothelial damage (1). Oxidative stress plays a role in the pathogenesis of ALI. Despite major advances in our understanding of the mechanisms leading to ALI, this condition remains a problem.

Lung alveolar interstitial fibroblasts and their communication with adjacent epithelial cells are important in lung development and injury/repair (2). The molecular mechanisms responsible for alterations of ALI have been extensively studied and a number of signaling pathways, including the JAK/STAT $(3,4)$, PI3 kinase/Akt $(5,6)$ and mitogen-activated protein kinase pathways (7-9), have been demonstrated to be important under a number of conditions.

The SHH signaling pathway consists chiefly of Shh, Ptc1, Smo, Gli1, Gli2 and Gli3 molecules. It has long been recognized that this pathway is essential for embryonic development, and has been revealed to regulate cell migration, proliferation and apoptosis (10). This cascade is crucial for the patterning of early lung morphogenesis (11). However, its role in acute lung injury/repair remains to be determined, particularly, the activation of the SHH signaling pathway which has been associated with hyperoxia. In the present study, we demonstrated that two significant molecules of this signaling pathway, Shh and its receptor Ptc1, are markedly activated in hyperoxia conditions as demonstrated by histomorphological changes and expression of genes and proteins.

\section{Materials and methods}

Animal model and treatment. All animal research was conducted following Home Office and Chongqing Medical University ethics committee approval. Animals received care according to the 'Guide for the Care and Use of Laboratory Animals' of the Institute of Laboratory Animal Research, Commission on Life Sciences. This study also followed the Institutional and National Institutes of Health Guidelines for Laboratory Animal Care.

Timed-pregnant Sprague-Dawley rats (provided by the Experimental Animal Center of Chongqing Medical University, Chongqing, China) were housed in individual 
cages with free access to water and laboratory food, and the rat pups were delivered spontaneously. Neonatal rats were nested on softwood shavings and distributed in litters of 10 of equal body weight in Plexiglass chambers. The chambers were equipped with a flow-through system for controlling the delivery of either medical oxygen or room air. Food and water were made available ad libitum, and lighting was provided on a 12-h light-dark cycle. Room and chamber temperature were maintained at $22-24^{\circ} \mathrm{C}$.

The experiment commenced within $6 \mathrm{~h}$ after birth and lasted until postnatal day 14 (P14d). Rat pups were randomly divided into two groups: hyperoxia group (HG) and normoxia group (NG). The groups were assigned to receive high oxygen concentration and room air, respectively.

In the $\mathrm{HG}, 6 \mathrm{l} / \mathrm{min}$ of medical oxygen flowed through the chambers, and the oxygen concentration was monitored continuously using an oxygen monitor and maintained at $95 \pm 2 \%$. In the NG, the oxygen concentration of room air in the chambers was maintained at $21 \pm 2 \%$. Carbon dioxide concentrations were maintained at $0.3 \%$, and relative humidity between 60 and $80 \%$. To avoid maternal oxygen toxicity, mother rats were rotated daily between the hyperoxic and room air chambers.

Survival and body weight of the rat pups in each group were assessed daily throughout the experiment. The rat pups were sacrificed at P3d, P7d and P14d; approximately six to eight animals at different times in each group were used. Under deep pentobarbital anesthesia $(50 \mathrm{mg} / \mathrm{kg}$, intraperitoneal), a midline incision was made through the sternum and abdomen, and whole-lung tissue was obtained. Lung and body weights were recorded at the time of sacrifice (12-14).

The left lungs were excised and fixed by overnight immersion in $4 \%$ paraformaldehyde in PBS at $4^{\circ} \mathrm{C}$. The specimens were dehydrated in a graded ethanol series and embedded in paraffin. The right lungs were resected after perfusion with ice-cold PBS, snap-frozen in liquid nitrogen and stored at $-80^{\circ} \mathrm{C}$ for subsequent biochemical analyses.

Histological examination. Sections $(5 \mu \mathrm{m})$ were cut from the paraffin blocks and stained with H\&E for general morphology. They were then examined under light microscopy, and the images were captured using a digital magnifier camera through an Olympus BX40 microscope (Olympus Optical, Tokyo, Japan). The Murakami technique was employed to determine the grade degree of lung injury (15) assessed according to histological features including edema, congestion, hemorrhage, infiltration of inflammatory cells and proliferation. Each of these features was graded as: 0 , absent and appears normal; 1, slight; 2 , moderate; 3 , strong; 4 , intense. A total score was calculated for each animal. To standardize the analyses, lung sections were collected from central areas of the superior lobe of the left lung, and morphometric analysis of each section was conducted in a blinded fashion by two independent observers.

Immunohistochemistry. Immunohistochemical staining for Shh and Ptcl was performed on paraffin sections using immunoperoxidase visualization. After deparaffinization, the sections were preincubated for $1 \mathrm{~h}$ at room temperature in $0.1 \mathrm{M}$ PBS containing $10 \%$ normal goat serum to block nonspecific binding of antibody, and immersed in $3 \% \mathrm{H}_{2} \mathrm{O}_{2}$ for $10 \mathrm{~min}$ to quench the endogenous peroxidase activity.
The tissue sections were then incubated overnight at $4^{\circ} \mathrm{C}$ with the following antibodies: anti-rat Shh rabbit antibody (1:100) and anti-rat Ptc1 rabbit antibody (1:100) (Abbiotec Biotechnology, Inc., San Diego, CA, USA). The tissue sections incubated with non-immune IgG were used as the negative controls. Secondary antibodies were obtained from the goat SABC-POD kit and visualized with the use of a DAB kit (Wuhan Boster Biological Technology Ltd., Wuhan, China) according to the manufacturer's instructions. Finally, the tissue slices were dehydrated in xylene and permanently mounted. Sections were examined and images were captured under the microscope using a digital magnifier camera.

RT-PCR for Shh and Ptcl mRNA. SHH and Ptcl mRNA was detected by RT-PCR. Lung tissue was ground into a powder in liquid nitrogen and the gene expression was measured using RT-PCR. The total RNA in the sample was extracted using an RNA TRIzol kit (Invitrogen Life Technologies, Paisley, UK) according to the manufacturer's instructions. Template cDNAs were obtained by reverse transcription of total RNAs using Oligo(dT) primer and Superscript II reverse transcriptase (Takara, Otsu, Japan). Amplification was conducted using SYBR Green QPCR Master mix (Takara). The expression level of $\beta$-actin was used as the internal control. The PCR primers for Shh, Ptcl and $\beta$-actin were designed and synthesized by Shinegene Molecular Biotech, Inc. (Shanghai, China). The sequences of the primers used were as follows: Shh forward, 5'TCGTGCTACGCAGTCATCG3'; reverse, 5'CGCTTC CGCTACAGATTGC3'; Ptc1 forward, 5'TGTGGCAACAGG ACGGAAC3'; reverse, 5'CCAGAGTGTCAGCAGAAGAA AAG3'; $\beta$-actin forward, 5'CCCATCTATGAGGGTTACGC3'; reverse, 5'TTTAATGTCACGCACGATTTC3'. All RT-PCR reactions were performed using an FTC2000 machine (Funglyn Biotech Inc., Scarborough, ON, Canada) using the following thermocycler conditions: 1 cycle at $94^{\circ} \mathrm{C}$ for 4 min; 35 cycles at $94^{\circ} \mathrm{C}$ for $20 \mathrm{sec}, 60^{\circ} \mathrm{C}$ for $30 \mathrm{sec}$, and $72^{\circ} \mathrm{C}$ for $30 \mathrm{sec}$. $\beta$-actin was used for each test sample along with the target genes. Gene expression was quantitatively analyzed using the comparative $\mathrm{Ct}\left({ }^{\Delta} \mathrm{Ct}\right)$ method, in which $\mathrm{Ct}$ was the threshold cycle number. For the target genes, Shh and Ptc1 mRNA levels were calculated using the following formula (16):

${ }^{\Delta \Delta} \mathrm{Ct}=$ Experimental group $\left(\mathrm{Ct}_{\text {target gene }}-\mathrm{Ct}_{\beta \text {-actin }}\right)-$ Control group $\left(\mathrm{Ct}_{\text {target gene }}-\mathrm{Ct}_{\beta \text {-actin }}\right)$,

where $2^{-\Delta \Delta \mathrm{Ct}}$ equals the amount of the target. Finally, the formula $2^{-\Delta \Delta C T}$ was used to calculate the amount of target RNA compared with the control.

Western blot analysis for detection of Shh and Ptcl proteins. The expression of Shh and Ptcl proteins was analyzed using western blot analysis. Frozen lung tissue was homogenized using a tissue grinder in ice-cold lysis buffer (RIPA buffer) containing $50 \mathrm{mM}$ Tris $\cdot \mathrm{HCl}(\mathrm{pH}$ 7.5), $1 \mathrm{mM}$ EGTA, $1 \mathrm{mM}$ EDTA supplemented with $1 \mathrm{mM}$ PMSF, phosphatase inhibitor and complete proteinase inhibitor cocktail (Sigma, St. Louis, MO, USA). The samples were sonicated and then centrifuged at $500 \mathrm{x} \mathrm{g}$ for $20 \mathrm{~min}$ at $4^{\circ} \mathrm{C}$ to remove cellular debris. Fifty micrograms of total protein for each sample was denatured by SDS-PAGE sample buffer and electrophoresed in a $10 \%$ SDS polyacrylamide gel. Resolved samples were then transferred onto PVDF membranes (Immobilon-P; Millipore, Bedford, 
Table I. Survival rate and lung/body weight in the hyperoxia and normoxia groups.

\begin{tabular}{lccccccc}
\hline & \multicolumn{3}{c}{ Survival rate $(\%)$} & & \multicolumn{3}{c}{ Lung/body weight ratio } \\
\cline { 2 - 3 } Group & P3d & P7d & P14d & & P3d & P7d & P14d \\
\hline HG & $94 \pm 10.0$ & $93 \pm 6.1$ & $72 \pm 7.3^{\mathrm{a}}$ & & $2.03 \pm 0.22$ & $1.69 \pm 0.19^{\mathrm{a}}$ & $1.83 \pm 0.24^{\mathrm{a}, \mathrm{b}}$ \\
NG & $100 \pm 0$ & $100 \pm 0$ & $100 \pm 0$ & & $1.91 \pm 0.26$ & $1.52 \pm 0.21$ & $1.33 \pm 0.24$ \\
\hline
\end{tabular}

Values are the mean \pm SEM. ${ }^{\text {a }}<0.05$ compared with $\mathrm{NG}$ at each time point; ${ }^{b} \mathrm{P}<0.05$ compared with postnatal day 7 (P7d). HG, hyperoxia group; NG, normoxia group.

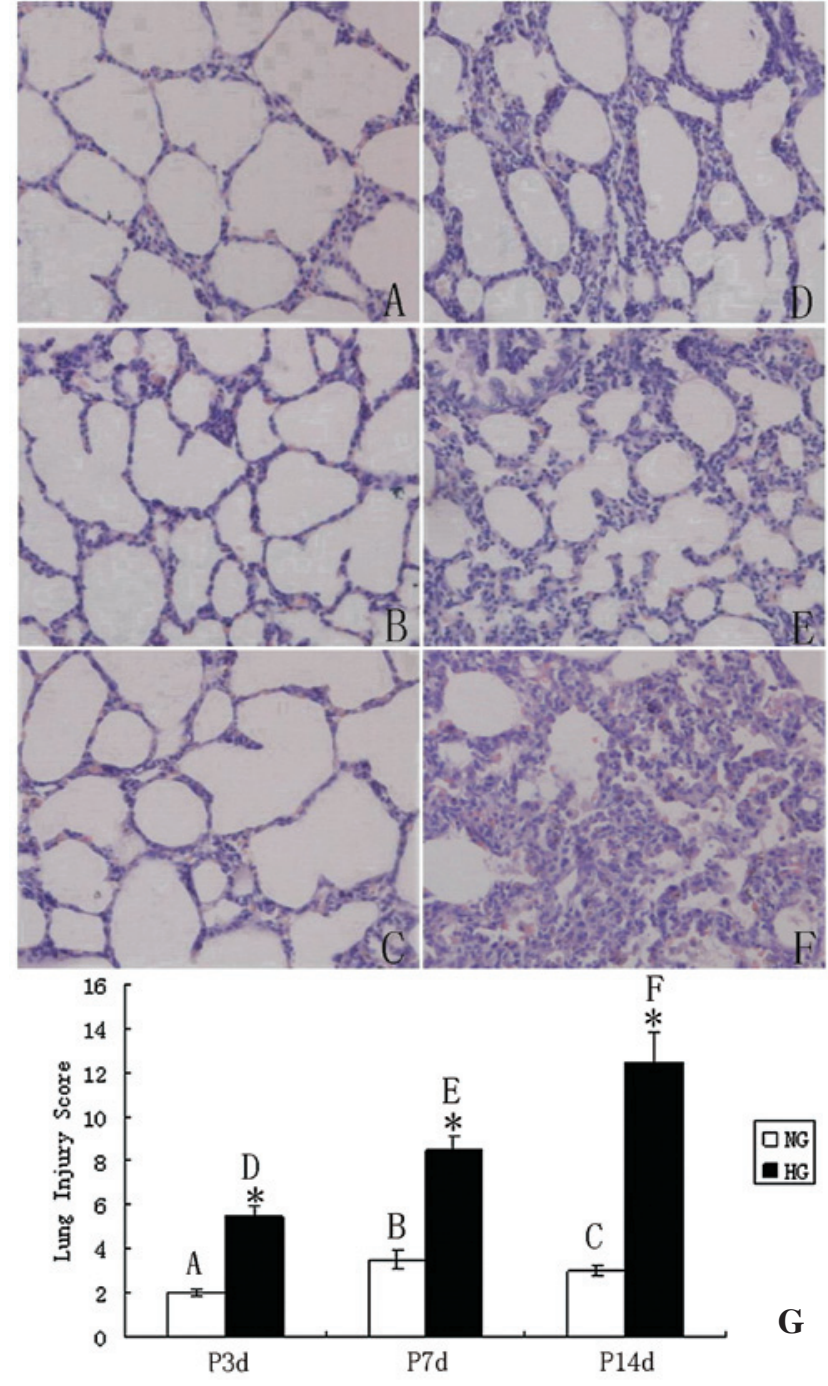

Figure 1. Morphological alterations of the lungs were determined using photomicrography. Photomicrographs of a pulmonary section from a rat pup in the (A-C) NG and (D-F) HG at postnatal day 3 (P3d), P7d and P14d, respectively. (G) Histopathological scoring of the NG and HG at each time point. Data are expressed as the mean \pm SEM $(n=6)$ and compared by the Kruskal-Wallis one way analysis of variance on ranks and the LSD method: ${ }^{*} \mathrm{P}<0.05$ compared with the NG group. Magnification, $\mathrm{x} 100$. HG, hyperoxia group; NG, normoxia group.

MA, USA), which, after blocking with TBS-Tween (TBST) and $5 \%$ milk, were incubated with the primary antibodies, anti-rat Shh (1:100) and Ptc1 (1:100) rabbit antibody or anti- $\beta$ - actin $\left(1: 1,000\right.$; Sigma) overnight at $4^{\circ} \mathrm{C}$, followed by the addition of horseradish peroxidase-conjugated goat anti-rabbit IgG or anti-rat IgG. Photographic film was used to capture protein bands, and densitometric analysis was performed to measure the intensity of the bands using the Quantity One 4.6 software program (Bio-Rad Laboratories, Hercules, CA, USA). Protein band intensities were normalized for loading using the corresponding GAPDH signals and expressed as arbitrary units (AU).

Statistical analysis. Experiments were performed at least 3 times independently. Each data point represents the mean of at least three parallel samples. The data are expressed as mean \pm SEM. Differences between the groups were evaluated using the one-way ANOVA test followed by the Fisher's least significant difference (LSD) post-hoc test and the unpaired Student's t-test as required. All data were analyzed using the SPSS statistical software (16.0 for Windows; SPSS Inc, Chicago, IL, USA). $\mathrm{P}<0.05$ was considered to indicate a statistically significant difference.

\section{Results}

Survival rate and lung/body weight ratio. Exposure of oxygen (HG) decreased the survival rate of the rat pups; the decline was most significant at $\mathrm{P} 14 \mathrm{~d}$ compared to the $\mathrm{NG}(\mathrm{P}<0.05)$. The lung/body weight ratio gradually decreased in the NG and the ratios in the $\mathrm{HG}$ were significantly greater than the ratios in the NG at $\mathrm{P} 7 \mathrm{~d}$ and $\mathrm{P} 14 \mathrm{~d}(\mathrm{P}<0.05)$, but in the HG the ratio increased at $\mathrm{P} 14 \mathrm{~d}$ compared with the value at $\mathrm{P} 7 \mathrm{~d}(\mathrm{P}<0.05)$ (Table I).

Lung histopathology. According to the H\&E-stained images, edema-like formation, neutrophil filtration, congestion, hemorrhaging and proliferation were evident in the HG compared with the NG. The degree of these histopathological changes in the lung became increasingly evident with prolonged hyperoxia. At P14d, the lungs of the hyperoxia-exposed pups had fewer secondary septa, wider interstitium inflammatory cell recruitment and fibrosis. Representative photomicrographs demonstrating differences in each experimental group are shown in Fig. 1.

Immunohistochemistry. In the $\mathrm{HG}$ at P7d and P14d, the Shh and Ptc1 proteins were observed in bronchial epithelial, alveolar epithelial and vascular endothelium cells, and were partly demonstrated in fibrotic tissue, fibroblasts and macro- 


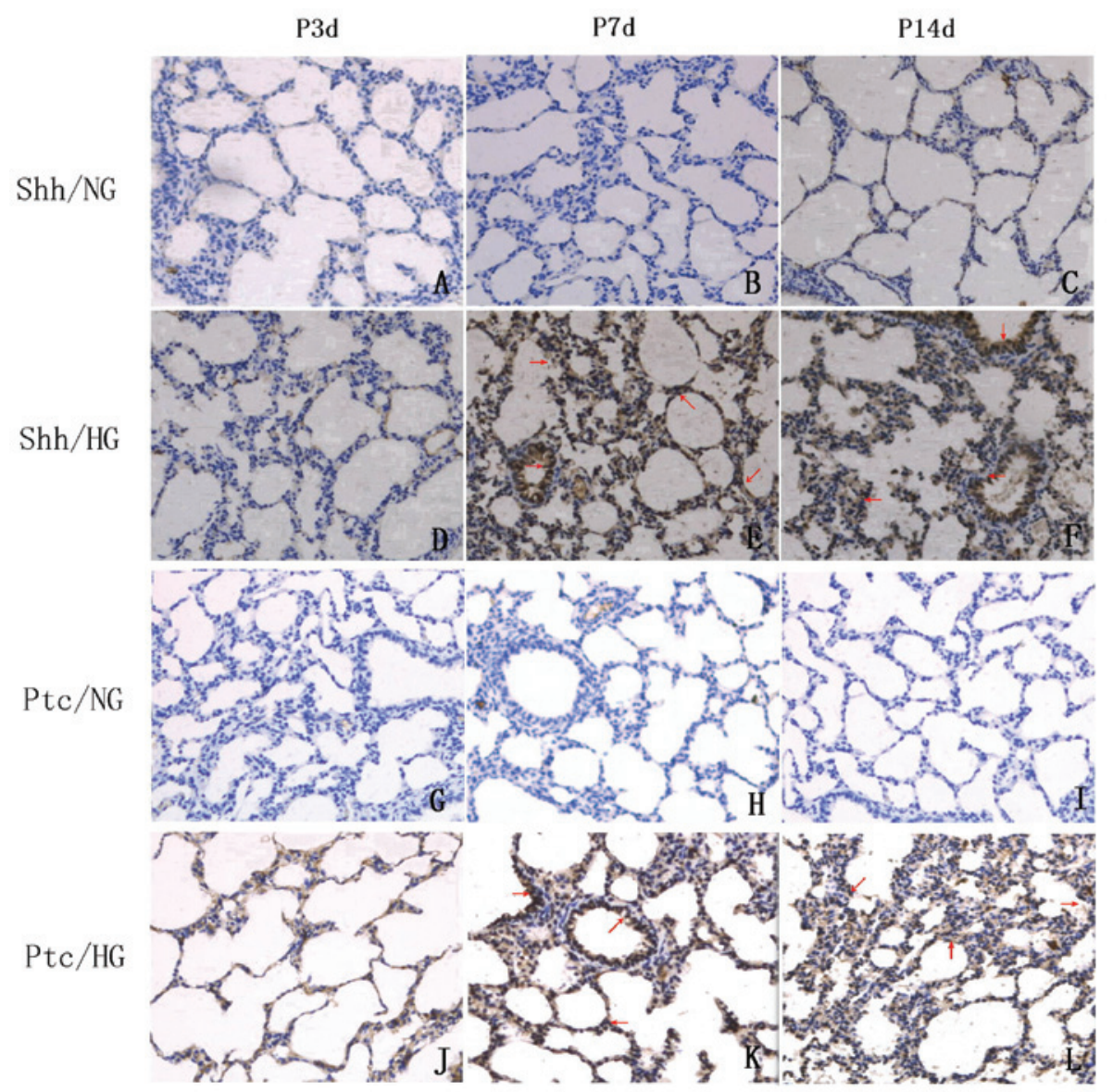

Figure 2. Immunohistochemical staining for Shh and Ptc1 in the lung sections from the normoxia- and hyperoxia-exposed neonatal rats (magnification, $\mathrm{x} 100$ ). Positive staining is demonstrated as brown (arrow). Photomicrographs at (A, D, G and J) postnatal day 3 (P3d), (B, E, H and K) P7d and (C, F, I and L) P14d. Rats exposed to (A-C, G-I) normoxia and (D-F, J-L) hyperoxia. Shh, sonic hedgehog; HG, hyperoxia group; NG, normoxia group.

A

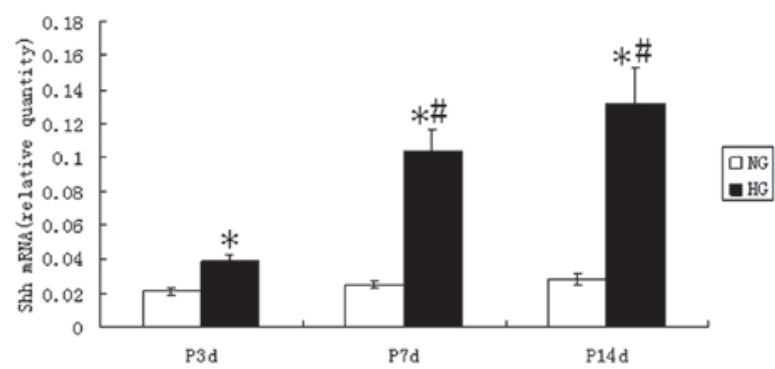

B

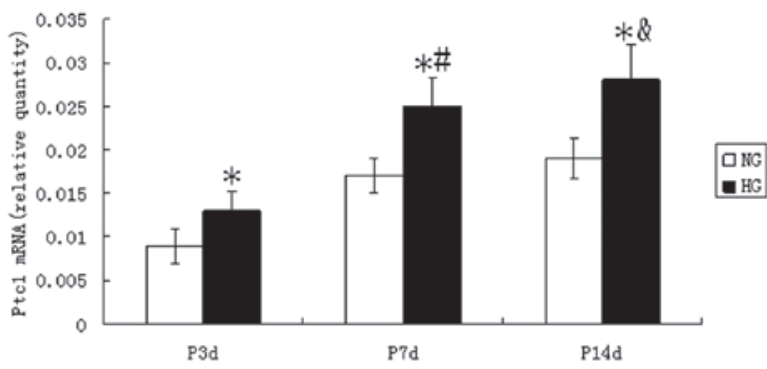

Figure 3. mRNA expression of Shh and Ptc-1 in rat pup lungs. (A) Shh mRNA expression was significantly higher in the HG than in the NG ("P<0.005; HG vs. NG). (B) Ptc1 mRNA expression was significantly higher in the HG than in the NG ("P<0.005; HG vs. NG). Shh and Ptc1 mRNA expression increased with time exposure to hyperoxia. Expression levels of Shh and Ptc-1 mRNA increased markedly at postnatal day 7 (P7d) compared to levels at P3d $\left({ }^{*} \mathrm{P}<0.05\right)$. Shh increased at $\mathrm{P} 14 \mathrm{~d}$ compared to $\mathrm{P} 7 \mathrm{~d}\left({ }^{*} \mathrm{P}<0.05\right)$; but compared with P7d, Ptc1 exibited no marked change at P14d ( $\left.{ }^{\&} \mathrm{P}<0.05\right)$. Shh, sonic hedgehog; HG, hyperoxia group; NG, normoxia group. phages in the lung interstitium. Weak Shh immunoreactivity was detected in the NG at P4d, but was not detected at P3d and P7d. Very weak or no anti-Ptc staining was detected for all times points in the NG. In the hyperoxia-exposed rats, Shh immunoreactivity increased on P7d, compared with the NG, and reached maximal levels at P14d. Ptc1 expression increased following treatment with hyperoxia for 7 days, but was lower than Shh and this continued to P14d (Fig. 2).

mRNA expression of Shh and Ptcl. Based on RT-PCR a significantly increased mRNA level of Shh was observed in the HG compared to the NG at the same time points. With increasing exposure to hyperoxia, Shh mRNA expression demonstrated a marked time-dependent increase in the hyperoxia-exposed rats $(\mathrm{P}<0.05)$. However, in regards to the Ptc1 mRNA, there was no statistically significant difference between P14 and P7d $(\mathrm{P}<0.05)$. The time-course effects of hyperoxia on Shh and Ptc1 mRNA expression in the lung at P3d, P7d and P14d are shown in Fig. 3.

Protein expression of Shh and Ptcl. We next examined the protein expression of Shh and Ptc1 in the lungs of the neonatal rats under hyperoxia and normoxia condtions. Significantly higher levels of Shh and Ptcl protein were noted following hyperoxia treatment, and the mean value was markedly greater 
A
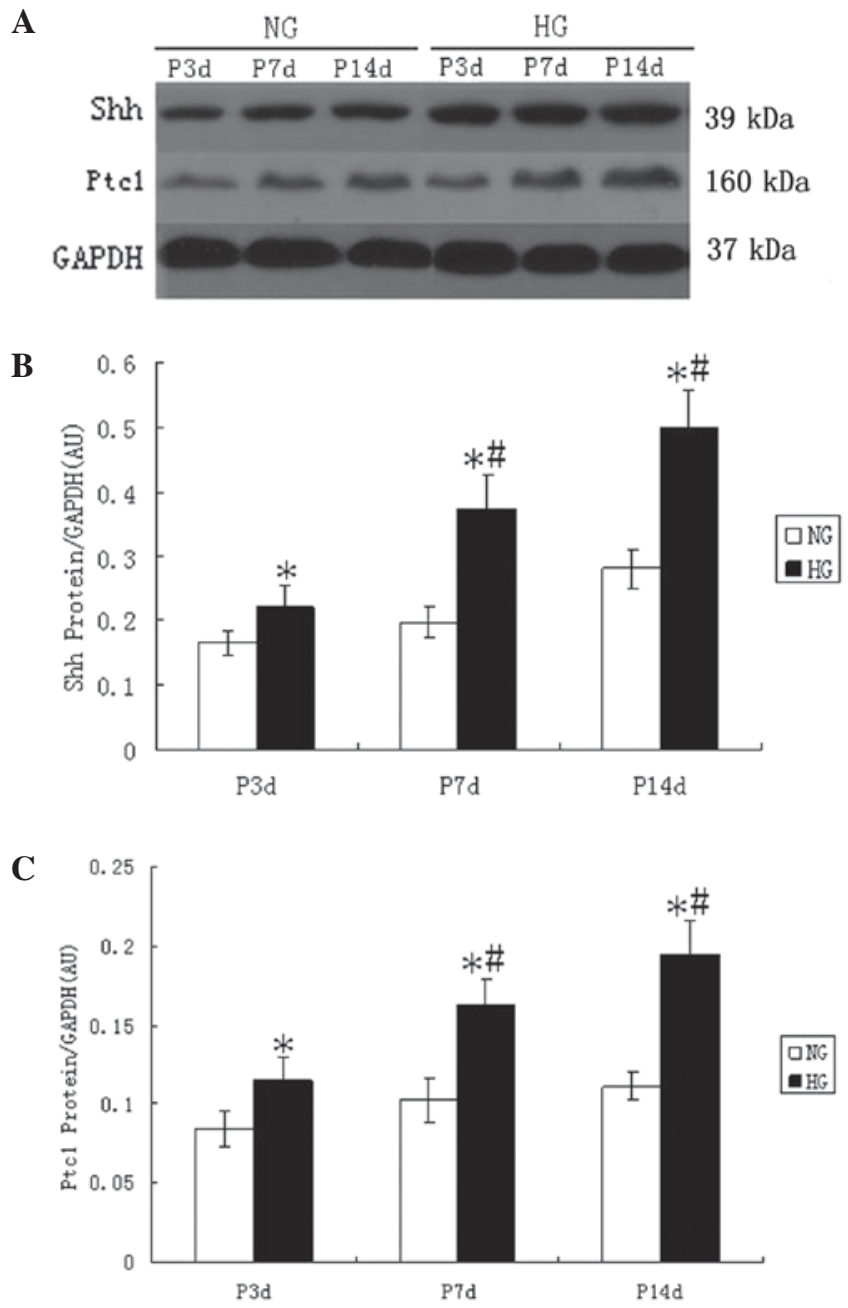

Figure 4. (A) Representative western blot analysis of the Shh and Ptc1 proteins in normoxia- (left lanes) and hyperoxia- (right lanes) exposed pup rat lungs. The significantly higher levels were observed in the HG compared to in the NG. (B and C) Quantitative data were determined by densitometry, and normalized to GADPH for each animal. Bars indicate the mean \pm SEM $(\mathrm{n}=6)$. Shh and Ptc1 protein levels increased following hyperoxia compared to normoxia treatment at each time point $\left({ }^{*} \mathrm{P}<0.05, \mathrm{HG}\right.$ vs. NG), and the mean value was significantly greater in the hyperoxia-exposed rats on postnata day 7 (P7d) and P14d when compared with rats on P3d, $\left({ }^{*} \mathrm{P}<0.05\right.$, P7d vs. P3d; P14d vs. P7d). The mean value reached a peak on P14d. Shh, sonic hedgehog; HG, hyperoxia group; NG, normoxia group.

in the hyperoxia-exposed rats on $\mathrm{P} 3 \mathrm{~d}, \mathrm{P} 7 \mathrm{~d}$ and $\mathrm{P} 14 \mathrm{~d}(\mathrm{P}<0.05)$. At P14d the mean value reached a peak. Western blot analyses demonstrated that the Shh and Ptc1 protein expression levels were significantly increased with a prolonged hyperoxia exposure time; Shh and Ptc1 were upregulated gradually in hyperoxia ( $\mathrm{P}<0.05$ vs. NG; Fig. 4).

\section{Discussion}

Therapy with hyperoxia is often required to treat newborns with respiratory disorders. However, supplemental oxygen administered to newborn infants with respiratory failure may increase oxidative stress and may lead to lung epithelial cell death in the immature lung, which is a well-known risk factor for the development of BPD $(17,18)$. Neonatal rodents are recognized to be tolerant to high levels of hyperoxia compared to adults. Recently, hyperoxia-induced pulmonary molecular changes in the neonatal rat BPD model have been described; murine alveolar development begins on P4d and saccular division is completed by P14d (13). During the 2-week period of hyperoxia in this study, the survival rate of the hyperoxiaexposed rats was reduced to $73 \%$, and the lung weight was reduced to a lesser extent than the body weight. We identified that hyperoxia-exposed rats exhibited significantly lower lung/ body weight ratios on $\mathrm{P} 7 \mathrm{~d}$ and higher lung/body weight ratios on P14d. We speculate that these findings were due to arrested lung development on P7d and increased lung inflammation or lung water content on P14d.

In the present study, lung development and inflammation were further supported by prominent histological alterations (19). Our in vivo model demonstrated that exposure of neonatal rats to hyperoxia caused lung water content, disruption of lung architecture and accumulation of inflammatory cells, compared to those under normoxia. With prolonged hyperoxia, the degree of these pathological features became increasingly evident; the survival rates of the rat pups were decreased and the lung/body weight ratio was altered. These phenomena are consistent with hyperoxia-induced lung injury.

The SHH pathway is a critically important developmental signaling system that regulates cell proliferation, differentiation, branching morphogenesis and patterning of mesenchyme (20). If the specific regulatory pathways are overexpressed or the inhibitory controls are blocked, dysregulated proliferation and tumorigenesis in the lung could have occurred (21). In certain organs (heart, liver, biliary tract, ovary, skin and prostate), SHH pathway dysregulation leads to hyperproliferation and tumorigenesis (22-26).

Shh, recognized to be expressed at low levels in the normal lung, is enhanced during repair of the damaged airway epithelium, in lung fibrotic diseases and fibrosis-associated inflammatory processes $(27,28)$. However, little is known concerning its function and importance in ALI and BPD, and it has not been studied under normal and hyperoxia conditions in neonatal rats.

In this study, changes in the molecular constituents of this pathway, Shh and its receptor Ptc1, were readily detected using standard biochemical assays, and the findings support substantial changes in expression levels. Shh and Ptc1 increased in response to hyperoxia, but at different time points. Shh mRNA was increased by P3d, and Ptc1 mRNA was increased by P7d following hyperoxia in the the lungs of rat pups. The proteins of these two signal molecules also demonstrated similar results. However, expression of these two molecules are weak in the normal lung. Concerning pathological changes, bronchial, alveolar epithelial and vascular endothelium cells were significantly damaged by hyperoxia; these changes could have occurred and improved in conjunction with increased levels of molecular constituents of the SHH signaling pathway in the first P14d. Thus, this pathway is likely activated during hyperoxia and is involved in the pathogenesis of hyperoxia-induced lung injury.

Shh and Ptc1 demonstrate an increasing trend during hyperoxia (HG) with a wide spectrum of expression in the lung. The main findings of this study demonstrate that the development of hyperoxia-induced lung injury was associated with increases in lung Shh and Ptc1 mRNA and protein expression. 
Upregulation of Shh and Ptcl expression preceded an increase in levels of lung pathological change; thus, an anti-SHH and Ptc1 strategy may attenuate lung injury and fibrosis. Further investigation of the role of Shh and Ptcl may offer new treatment strategies for hyperoxia-induced lung injury. However, there were a number of limitations in this study. The full spectrum of mechanisms of the Shh signaling pathway in hyperoxia-induced ALI and BPD are not completely understood. An effective clinical solution remains to be developed. Further investigation is necessary to develop highly selective pharmacological agents and other novel strategies, including Shh and Ptcl antagonists or Shh-knockout animal models for wide-scale employment by clinicians.

\section{Acknowledgements}

This study was supported by the National Natural Science Foundation of China (No. 81071573).

\section{References}

1. Altemeier WA and Sinclair SE: Hyperoxia in the intensive care unit: why more is not always better. Curr Opin Crit Care 13: 73-78, 2007.

2. Demayo F, Minoo P, Plopper CG, Schuger L, Shannon J and Torday JS: Mesenchymal-epithelial interactions in lung development and repair: are modeling and remodeling the same process? Am J Physiol Lung Cell Mol Physiol 283: 510-517, 2002

3. Severgnini M, Takahashi S, Rozo LM, et al: Activation of the STAT pathway in acute lung injury. Am J Physiol Lung Cell Mol Physiol 286: 1282-1292, 2004.

4. Severgnini M, Takahashi S, Tu P, et al: Inhibition of the Src and Jak kinases protects against lipopolysaccharide-induced acute lung injury. Am J Respir Crit Care Med 171: 858-867, 2005.

5. Tejera P, Wang Z, Zhai R, et al: Genetic polymorphisms of peptidase inhibitor 3 (elafin) are associated with acute respiratory distress syndrome. Am J Respir Cell Mol Biol 41: 696-704, 2009.

6. Yum HK, Arcaroli J, Kupfner J, et al: Involvement of phosphoinositide 3-kinases in neutrophil activation and the development of acute lung injury. J Immunol 167: 6601-6608, 2001.

7. Schuh K and Pahl A: Inhibition of the MAP kinase ERK protects from lipopolysaccharide-induced lung injury. Biochem Pharmacol 77: 1827-1834, 2009.

8. Moon C, Han JR, Park HJ, Hah JS and Kang JL: Synthetic RGDS peptide attenuates lipopolysaccharide-induced pulmonary inflammation by inhibiting integrin signaled MAP kinase pathways. Respir Res 10: 18, 2009.

9. Damarla M, Hasan E, Boueiz A, et al: Mitogen activated protein kinase activated protein kinase 2 regulates actin polymerization and vascular leak in ventilator associated lung injury. PLoS One 4: e4600, 2009.

10. Watson S, Serrate C and Vignot S: Sonic hedgehog signaling pathway: from embryology to molecular targeted therapies. Bull Cancer 97: 1477-1483, 2010.
11. Jacob L and Lum L: Deconstructing the hedgehog pathway in development and disease. Science 318: 66-68, 2007.

12. Chang YS, Kim YJ, Yoo HS, et al: Alpha-phenyl-N-tertbutylnitrone attenuates hyperoxia-induced lung injury by down-modulating inflammation in neonatal rats. Exp Lung Res 35: 234-249, 2009.

13. Tao W, Shu YS, Miao QB and Zhu YB: Attenuation of hyperoxiainduced lung injury in rats by adrenomedullin. Inflammation 35: 150-157, 2011.

14. Husari AW, Dbaibo GS, Bitar H, et al: Apoptosis and the activity of ceramide, Bax and Bcl-2 in the lungs of neonatal rats exposed to limited and prolonged hyperoxia. Respir Res 7: 100, 2006.

15. Murakami K, McGuire R, Cox RA, et al: Heparin nebulization attenuates acute lung injury in sepsis following smoke inhalation in sheep. Shock 18: 236-241, 2002.

16. Livak KJ and Schmittgen TD: Analysis of relative gene expression data using real-time quantitative PCR and the 2(-Delta Delta C(T) method. Methods 25: 402-408, 2001.

17. Saugstad OD: Bronchopulmonary dysplasia-oxidative stress and antioxidants. Semin Neonatol 8: 39-49, 2003.

18. Spiteller G: The important role of lipid peroxidation processes in aging and age dependent diseases. Mol Biotechnol 37: 5-12, 2007.

19. Kunig AM, Balasubramaniam V, Markham NE, Seedorf G, Gien J and Abman SH: Recombinant human VEGF treatment transiently increases lung edema but enhances lung structure after neonatal hyperoxia. Am J Physiol Lung Cell Mol Physiol 291: 1068-1078, 2006.

20. Mill P, Mo R, Fu H, et al: Sonic hedgehog-dependent activation of Gli2 is essential for embryonic hair follicle development. Genes Dev 17: 282-294, 2003.

21. Karhadkar SS, Bova GS, Abdallah N, et al: Hedgehog signalling in prostate regeneration, neoplasia and metastasis. Nature 431: 707-712, 2004.

22. Kusano KF, Pola R, Murayama T, et al: Sonic hedgehog myocardial gene therapy: tissue repair through transient reconstitution of embryonic signaling. Nat Med 11: 1197-1204, 2005.

23. Lavine KJ, Kovacs A and Ornitz DM: Hedgehog signaling is critical for maintenance of the adult coronary vasculature in mice. J Clin Invest 118: 2404-2414, 2008.

24. Omenetti A, Yang L, Li YX, et al: Hedgehog-mediated mesenchymal-epithelial interactions modulate hepatic response to bile duct ligation. Lab Invest 87: 499-514, 2007.

25. Sicklick JK, Li YX, Melhem A, et al: Hedgehog signaling maintains resident hepatic progenitors throughout life. Am J Physiol Gastrointest Liver Physiol 290: G859-G870, 2006.

26. Wetmore C: Sonic hedgehog in normal and neoplastic proliferation: insight gained from human tumors and animal models. Curr Opin Genet Dev 13: 34-42, 2003.

27. Stewart GA, Hoyne GF, Ahmad SA, et al: Expression of the developmental Sonic hedgehog (Shh) signalling pathway is up-regulated in chronic lung fibrosis and the Shh receptor patched 1 is present in circulating T lymphocytes. J Pathol 199: 488-495, 2003.

28. Watkins DN, Berman DM, Burkholder SG, Wang B, Beachy PA and Baylin SB: Hedgehog signalling within airway epithelial progenitors and in small-cell lung cancer. Nature 422: 313-317, 2003. 